\title{
Artemisinin and its Derivatives as Repurposing Drug against COVID-19
}

\section{Kanchan Kumari and Sandip K Mishra*}

Cancer Biology Laboratory, Department of Cancer Biology, Institute of Life Sciences (Department of Biotechnology, Govt of India), Bhubaneswar, Odisha, India

*Corresponding Author: Sandip K Mishra, Scientist-F, Cancer Biology Laboratory, Department of Cancer Biology, Institute of Life Sciences, Bhubaneswar, Odisha, India.

DOI: $10.31080 /$ ASCB.2020.04.0237

Keywords: COVID-19; Artemisinin; Hydroxychloroquine

The world is experiencing the outbreak of highly infectious RNA virus named COVID-19 or SARS-CoV-2 since December last year. As on 28 May 2020, World Health Organization (WHO) has reported 5,556,679 confirmed cases of COVID-19, including 351,866 deaths worldwide. Clinicians and scientists now know much about the characteristics of the virus and its effect on human health which is similar to SARC-CoV but with more serious outcomes [1]. Taking the help of its own spike (S) glycoprotein and host's receptor protein called angiotensin converting enzyme 2 (ACE-2) present on the cell membrane, the SARS-CoV-2 virus enters in the cells and releases its genome that integrates into the host genome. After hijacking the host cell regulated system it replicates in large number, infecting nearby cells and leading the infection to spread in different organs of the body [2]. The immune system of host might be one of the factors that can explain disease presentation leaving some people asymptomatic and others severely affected [3]. The immune response induced by COVID-19 infection leads to high levels of proinflammatory cytokines, neutrophils and reduced number of total lymphocytes affecting both innate and acquired immunity $[4,5]$.

Hydroxychloroquine is one of the most promising drugs against COVID-19 [6] disease progression and management. Treatment with hydroxychloroquine show effective clearance of viral load in COVID-19 patients as reported in human trials $[7,8]$. Hydroxychloroquine is known as a potent anti-malarial drug also effective in disorders like Systemic lupus erythematosus (SLE), Cutaneous lupus erythematosus, Rheumatoid arthritis (RA) [9] and anti-SSA/ Ro-antibody-associated congenital heart disorder. As an immunomodulatory, reports suggest that hydroxychloroquine alters T cell responses by inhibiting cytokines, INF $\alpha$, TNF $\alpha$ and many more [10]. It inhibits Toll-like receptors 3, 7, 8 and 9 which results in reduced dendritic cell activation and reduced interferon production [11]. The inhibition of TLRs elucidates the function of hydroxychloroquine as a weak base leading to acidification of endosome [12]. Encouraging impact of hydroxychloroquine on glucose level [13] and in cardiac dysfunctions [14] explains its effectiveness against COVID-19, where diabetes [15] and cardiovascular disorders play critical role in disease onset and progression [16].

We have proved that the antimalarial drug, i.e. Artemisinin could work against Breast cancer metastasis [17]. Our hypothesis says that Artemisinin will work to fight COVID-19 infection. I would say that the way an anti-malarial drug i.e. hydroxychloroquine is showing great efficacy against COVID-19, Artemisinin is also going to be proven as an effective drug against COVID-19. New scientific discoveries come out of wild imagination but the imagination should be based on at least some reliable evidences, which may be direct or indirect. Artemisinin-based combination therapy [17] has been adopted by the World Health Organization as a first-line treatment for uncomplicated Plasmodium falciparum malaria. In endemic regions, it has proven more effective in treating the disease and even in reducing its transmission. Artemisinin is a chemical compound that reacts with iron to form free radicals, which can kill cells. Cancer cells require and uptake a large amount of iron to proliferate. They are more susceptible to the cytotoxic effect of artemisinin than normal cells.

Artemisinin and its derivatives represent the most important and influential class of drugs in the fight against malaria [18]. Since the discovery of Artemisinin in the early 1970s, the global community has made great strides in characterizing and understanding this remarkable phytochemical and its unique chemical and pharmacological properties. Today, even as Artemisinin continues to serve as the foundation for antimalarial therapy, numerous challenges have surfaced in the continued application and development of this family of drugs. These challenges include the emergence of delayed treatment responses to Artemisinin in malaria and efforts to apply Artemisinin for non-malarial indications. Here, we comment on the current understanding of the mechanism of action (MOA) of Artemisinin and in particular emphasize the importance of relating mechanistic studies to therapeutic outcomes, both in malarial and non-malarial contexts. 
Here we propose Artemisinin and its derivatives (dihydroartemisinin (DHA), artemether, and artesunate) as promising drug candidates for treatment of COVID-19. Artemisia annua derived Artemisinin a well-known anti-malarial drug [19] that show higher chemotherapeutic index than chloroquine and is reported to be effective even in chloroquine-resistant human malarial strains [20]. As per a recent findings, Artesunate, a derivative of Artemisinin exhibits greater anti-inflammatory effect than hydroxychloroquine in RA patients [21]. WHO has recommended Artemisinin and its synthetic derivatives as a first line treatment in malaria [22]. Similar to hydroxychloroquine, Artemisinin and its derivatives effectively modulate immune system and provide direct benefit to host system, which is well evidenced by several groups of researchers [2327]. Silva., et al. 2018 discusses the progress that have been made in delivery of Artemsinin and its derivatives for use for immunomodulatory purposes [28]. The substantial effect of Artemisinin and its derivatives on immune system can show promising results against immune related diseases [29]. Interestingly, encouraging immune suppressive results have been observed in combination therapy of Artemisinin and Hydroxychloroquine [30,31]. To our expectations, Artesunate shows strong anti-viral activities specially Hepatitis B virus and Hepatitis C virus as recently reported [32]. To address the availability of stable source of Artemisinin, efforts have been made for high-level semi-synthetic production of Artemisinin [33], which suggests the substantial efficacy of the drug and can be taken up in case of high demand.

In conclusion, immunomodulatory and anti-viral properties of Artemisinin and its derivatives may prove to be of great therapeutic importance against COVID-19 infection and disease progression.

\section{Bibliography}

1. Guan WJ., et al. "Clinical Characteristics of Coronavirus Disease 2019 in China”. The New England Journal of Medicine (2020).

2. Walls AC., et al. "Structure, Function, and Antigenicity of the SARS-CoV-2 Spike Glycoprotein". Cell (2020).

3. Rothe C., et al. "Transmission of 2019-nCoV Infection from an Asymptomatic Contact in Germany". The New England Journal of Medicine 382.10 (2020): 970-971.

4. Prompetchara E., et al. "Immune responses in COVID-19 and potential vaccines: Lessons learned from SARS and MERS epidemic". Asian Pacific Journal of Allergy and Immunology 38.1 (2020): 1-9.

5. Shi Y., et al. "COVID-19 infection: the perspectives on immune responses". Cell Death and Differentiation (2020).
6. Colson P., et al. "Chloroquine and hydroxychloroquine as available weapons to fight COVID-19". International Journal of Antimicrobial Agents (2020): 105932.

7. Gautret P., et al. "Hydroxychloroquine and azithromycin as a treatment of COVID-19: results of an open-label non-randomized clinical trial". International Journal of Antimicrobial Agents (2020): 105949.

8. Singh AK., et al. "Chloroquine and hydroxychloroquine in the treatment of COVID-19 with or without diabetes: A systematic search and a narrative review with a special reference to India and other developing countries". Diabetes and Metabolic Syndrome 14.3 (2020): 241-246.

9. Schrezenmeier E and Dorner T. "Mechanisms of action of hydroxychloroquine and chloroquine: implications for rheumatology". Nature Reviews Rheumatology 16.3 (2020): 155-166.

10. Van den Borne BE., et al. "Chloroquine and hydroxychloroquine equally affect tumor necrosis factor-alpha, interleukin 6, and interferon-gamma production by peripheral blood mononuclear cells". The Journal of Rheumatology 24.1 (1997): 55-60.

11. Durcan L and Petri M. "Immunomodulators in SLE: Clinical evidence and immunologic actions". Journal of Autoimmunity 74 (2016): 73-84.

12. Lafyatis R., et al. "Antimalarial agents: closing the gate on Tolllike receptors?" Arthritis and Rheumatism 54.10 (2006): 30683070 .

13. Gerstein HC., et al. "The effectiveness of hydroxychloroquine in patients with type 2 diabetes mellitus who are refractory to sulfonylureas--a randomized trial". Diabetes Research and Clinical Practice 55.3 (2002): 209-219.

14. Izmirly PM., et al. "Evaluation of the risk of anti-SSA/Ro-SSB/ La antibody-associated cardiac manifestations of neonatal lupus in fetuses of mothers with systemic lupus erythematosus exposed to hydroxychloroquine". Annals of the Rheumatic Diseases 69.10 (2010): 1827-1830.

15. Bloomgarden ZT. "Diabetes and COVID-19". Journal of Diabetes 12.4 (2020): 347-348.

16. Zheng YY., et al. "COVID-19 and the cardiovascular system". Nature Reviews Cardiology (2020).

17. Acton N., et al. "Synthesis and antimalarial activity of some 9-substituted artemisinin derivatives". Journal of Medicinal Chemistry 36.17 (1993): 2552-2557. 
18. Jigang Wang CX., et al. "Artemisinin, the Magic Drug Discovered from Traditional Chinese Medicine". Engineering, ScienceDirect, Elsevier 5.1 (2019): 32-39.

19. Klayman DL. “Qinghaosu (artemisinin): an antimalarial drug from China”. Science 228.4703 (1985): 1049-1055.

20. Mojab F. "Antimalarial natural products: a review". Avicenna Journal of Phytomedicine 2.2 (2012): 52-62.

21. Ma JD., et al. "A novel function of artesunate on inhibiting migration and invasion of fibroblast-like synoviocytes from rheumatoid arthritis patients". Arthritis Research and Therapy 21.1 (2019): 153.

22. Organization WH. "Guidelines for the treatment of malaria". $3^{\text {rd }}$ edition. Geneva:(WHO Press) (2015).

23. Zhang T., et al. "Dihydroartemisinin regulates the immune system by promotion of CD8(+) T lymphocytes and suppression of B cell responses". Science China Life Sciences (2019).

24. Hou L and Huang H. "Immune suppressive properties of artemisinin family drugs". Pharmacology and Therapeutics 166 (2016): 123-127.

25. Ma RJ., et al. "Artemisinin and its derivatives: a potential therapeutic approach for oral lichen planus". Inflammation research: official journal of the European Histamine Research Society 68.4 (2019): 297-310.

26. Yang Z., et al. "Immunomodulatory and anti-inflammatory properties of artesunate in experimental colitis". Current Medicinal Chemistry 19.26 (2012): 4541-4551.

27. Li Z., et al. "Artemisinin and Its Derivatives as a Repurposing Anticancer Agent: What Else Do We Need to Do?" Molecules 21.10 (2016).

28. Charlie-Silva I., et al. "Progress in nano-drug delivery of artemisinin and its derivatives: towards to use in immunomodulatory approaches". Artificial Cells, Nanomedicine, and Biotechnology 46.3 (2018): S611-S620.

29. Wang Wyfwh. "Immunomodulation of artemisinin and its derivatives”. Science Bulletin 61.18 (2016): 1399-1406.

30. Bai L., et al. "Immunosuppressive effect of artemisinin and hydroxychloroquine combination therapy on IgA nephropathy via regulating the differentiation of CD4+ T cell subsets in rats". International Immunopharmacology 70 (2019): 313323.
31. Liang N., et al. "Immunosuppressive effects of hydroxychloroquine and artemisinin combination therapy via the nuclear factor-kappaB signaling pathway in lupus nephritis mice". Experimental and Therapeutic Medicine 15.3 (2018): 2436-2442.

32. D’Alessandro S., et al. "The Use of Antimalarial Drugs against Viral Infection". Microorganisms 8.1 (2020).

33. Paddon CJ., et al. "High-level semi-synthetic production of the potent antimalarial artemisinin". Nature 496.7446 (2013): 528-532.

\section{Assets from publication with us}

- Prompt Acknowledgement after receiving the article

- Thorough Double blinded peer review

- Rapid Publication

- Issue of Publication Certificate

- High visibility of your Published work

Website: https://www.actascientific.com/

Submit Article: https://www.actascientific.com/submission.php Email us: editor@actascientific.com

Contact us: +919182824667 